\title{
Entropy Generation Analysis of Human Thermal Stress Responses
}

\author{
Satish C. Boregowda, ${ }^{1}$ Robert E. Choate, ${ }^{2}$ and Rodney Handy ${ }^{3}$ \\ ${ }^{1}$ Sandia National Laboratories, P.O. Box 5800, Albuquerque, NM 87185, USA \\ ${ }^{2}$ Department of Engineering, Western Kentucky University, Bowling Green, KY 42101, USA \\ ${ }^{3}$ Department of Engineering Technology, University of North Carolina, Charlotte, NC 28223, USA \\ Correspondence should be addressed to Satish C. Boregowda, sborego@sandia.gov
}

Received 18 October 2012; Accepted 12 November 2012

Academic Editors: S. Hashimoto and M. Sanati

Copyright ( $\odot 2012$ Satish C. Boregowda et al. This is an open access article distributed under the Creative Commons Attribution License, which permits unrestricted use, distribution, and reproduction in any medium, provided the original work is properly cited.

The present study involves application of an open system entropy generation formulation to analyze human thermal stress responses. The time-series human thermal stress response data are obtained by conducting a simulation using a validated finiteelement human thermal model (FEHTM). These simulated human thermal response data are used as an input to the entropy generation expression to obtain human entropy generation (HEG) values. The effects of variables such as air temperature, relative humidity, physical activity, and clothing on entropy generation are examined. A design of experiment (DOE) approach is utilized to study the interaction effects of air temperature and relative humidity on entropy generation. The study establishes the importance and utility of entropy generation as a holistic measure of human thermal physiological reaction to external and internal changes. This novel study has great potential for use in military medicine, rehabilitation, sports, and related applications.

\section{Introduction}

Numerous studies have been conducted in the past to establish the fact that human beings are homeotherms, who continuously exchange energy, with their environment. In doing so, they maintain fairly constant internal temperatures, in spite of widely varying environmental conditions. This state of maintaining constant internal temperature is called homoeothermic state. For a healthy, sedentary human, core temperature is typically around $37.0^{\circ} \mathrm{C}$, measured rectally. Deviations of $2^{\circ} \mathrm{C}$ in core temperature from its normal level may cause discomfort. As core temperature rises above this narrow range, body functions begin to deteriorate. This condition is called hyperthermia. When the body temperature falls below the normal temperature range, a condition called hypothermia exists. The maintenance of a homoeothermic state is very important for the existence and support of life. Thus, the study of the response of homeotherms to environmental and internal thermal stresses occupies an important position in the fields of life sciences, indoor built environments, and engineering.

The thermoregulatory system is the physiological system responsible for maintaining the core temperature at a normal level by enhancing or inhibiting thermal energy production and loss. Thermoreceptors, located in the skin, brain, spinal cord, and other sites in the body, respond to both local temperature and its changes. Signals from the thermoreceptors are transmitted by the central nervous system to the hypothalamus where they are integrated in a manner which is not completely understood at this time. Regardless of the relative significance of the temperature signals from the various thermoreceptor locations, the hypothalamus determines the overall thermal state of the body from these signals and issues the appropriate effector commands. There are three types of thermoregulatory responses which either inhibit or enhance thermal energy production and loss: vasomotor, sudomotor, and metabolic. The body's response to dilate or constrict skin blood vessels is a vasomotor response where dilation of skin blood vessels is referred to as vasodilation and constriction of those same blood vessels is called vasoconstriction. When core temperature continues to rise despite the body's effort to return it to a normal level through vasodilation, a sudomotor response, commonly known as sweating, occurs. Finally, thermal energy production in humans arises from three sources: basal metabolism, voluntary physical activity, and shivering. 
The basal metabolic rate is the rate at which thermal energy is generated during the resting condition. The total rate of thermal energy production from these three sources is called the metabolic rate.

The present study involves the use of second-law of thermodynamics to better understand the dynamics of this human thermal activity. It is based on the earlier studies conducted by Aoki $[1,2]$. Aoki, a Japanese biophysicist has done pioneering work in applying the concept of secondlaw to calculate entropy production in the human body under basal and exercise conditions. This study, however, uses only static experimental data to establish the fact that entropy generation is positive in accordance with the second law. The present study overcomes this limitation by conducting a detailed parametric study with aid of dynamic simulation data by examining the effects of physical and personal variables on entropy generation. This parametric study provides a deeper insight into the utility of entropy generation for use in relevant industrial applications. Some of the applications are in the areas of architecture, built environment, military, and aviation.

The present simulation study is conducted with the help of a validated finite-element human thermal model (FEHMT) of Smith [3] and Fu [4]. Further, Boregowda and Tiwari [5] utilized FEHMT to evaluate thermal comfort in various space enclosures. These well-established models are used to conduct series of computer simulations to produce time-series data of human thermal responses to different environmental conditions. The output from the simulation is used as input to the entropy generation expression, which combines both human thermal physiological responses and environmental variables to compute human entropy generation.

\section{Modeling, Formulation, and Solution Methods}

2.1. Terminology. It is important to note that words such as "heat loss," "heat generation," and "heat transfer" are widely used in thermal physiology literature, which is consistent with the macroscopic nature of energetic interaction between humans and their surroundings. However, this terminology is not consistent with the concept of energy as defined by Gyftopoulos and Beretta [6]. In lieu of the word "heat," the word "thermal energy" will be used throughout this current thermophysiological study that primarily deals with macroscopic aspects of thermal transport mechanisms. The authors are aware of this inconsistency in the thermal physiology literature and will use thermodynamically consistent terms and expressions in this paper.

2.2. Review of Human Thermal Models. The thermoregulatory system regulates thermal energy production and loss through vasodilation, vasoconstriction, shivering, and sweating using temperature feedback from thermoreceptors located throughout the body [7]. Numerous efforts have been made in the past to develop models to better understand human thermal physiology, which are being reviewed here.
The human thermal models range from simple, onedimensional, steady-state simulations to complex, transient, finite element codes with thousands of nodes. Whether these models are simple or complex, they have limitations for use in evaluation of thermal comfort standards or similar uses. These limitations include the accuracy of the physical simulation and the accuracy of the inputs to the model. Perhaps, the biggest limitation is the accuracy with which comfort perceptions can be related to the physiological variables simulated in the thermal models [8]. One of the earliest models was developed by Givoni and Goldman [9], which represented the whole human body as a single node. This model is empirical and is applicable only to hot environments. The two-node model, later developed by Gagge et al. [10], separated the human body to core layer and skin surface layer and comprised of two subsystems: the control system and the passive system. This model was applicable for moderate activity levels and uniform environmental conditions, but it was limited to human exposure times of less than an hour and could not account for spatial non-uniformities. During same time period, Stolwijk [11] had developed a multinode model but it was limited to constant environmental conditions. In this model, the body was basically divided into six segments and each one was further divided into four layers in radial direction. Because the physiological phenomena of each section could be controlled, this model had better precision and flexibility than the previous ones. Even though the Stolwijk model was still limited to constant environment conditions, it was a milestone in the human thermal model development history. Most of subsequent contemporary multisegment multi-node bioheat models are modifications and improvements on Stolwijk model parameters and thermal control equations. Table 1 presents the most widely used human thermal physiological models ranging from the simplest one-node model to the complex three-dimensional finite element model [12].

Another notable two-node model was developed at the Institute for Environmental Research (IER) at the Kansas State University (KSU) by Azer and Hsu [13]. The twonode KSU model was similar to Stolwijk model but had different control equations to simulate sweating and blood flow. This earlier KSU two-node model was able to determine thermal sensation directly from physiological strain. In these earlier human thermal models, clothing insulation was either ignored or was treated as a single parameter that could be applied to the whole body. Consequently, Jones and Ogawa [15] have developed a transient model of heat and moisture transport through the clothing using relatively simple thermal models of the body. With the advances in computer hardware and software technology, the Smith-Fu model [3, 4] that uses a 3000-node finite element model was developed at KSU to simulate the clothed human body. This model has become the basis for National Renewable Energy Laboratory (NREL) studies on automotive interior and built environment energy conservation studies [18]. The other models listed in Table 1 include the ones by Fiala et al. [16], Huizenga et al. [17], and Tanabe et al. [19]. In summary, it is clearly stated by Jones [8] that human 
TABLE 1: Human thermal models.

\begin{tabular}{lcl}
\hline Date & Author & Description \\
\hline 1971 & Givoni and Goldman [9] & One-Node; Empirical \\
1971 & Gagge et al. [10] & Two-Node \\
1971 & Stolwijk [11] & Multi-Node \\
1977 & Azer and Hsu [13] & Two-Node \\
1985 & Wissler [14] & 225-Node Finite Element \\
1991 & Smith [3] & 3000-Node Finite Element 3D Transient \\
1992 & Jones and Ogawa [15] & Two-Node Clothed Transient \\
1995 & Fu [4] & 3000-Node Finite Element 3D Clothed Transient \\
1999 & Fiala et al. [16] & Multi-Node; 366 Tissue Nodes \\
2001 & Huizenga et al. [17] & Multi-Node Thermal Model; Arbitrary Number of Segments \\
1995 & Tanabe & Multi-Node; 65-Node \\
\hline
\end{tabular}

thermal physiological stress response modeling is somewhat less accurate. The actual energy balances on the body or body components are relatively straightforward once the heat and moisture exchanges with the environment and the metabolic heat generation are known. However, the thermoregulatory responses and person-to-person variations in stress response are not understood sufficiently that they can be modeled to exactly simulate what occurs in the body. Various empirical relationships are used in the models to relate factors such as sweat rate and blood flows to various body conditions. It is virtually impossible to directly verify these relationships and they can only be indirectly verified by comparing simulation results to physiological data. It is quite likely that most of the models use relationships that are in fact only surrogates for the true control variables in the body and/or the relationships are only crude representations of the relationships actually employed by the body. Thus, any model used in this kind of study is expected to show some discrepancy with experimental data. Due to the fact that Smith-Fu model $[3,4]$ is capable of modeling irregular shaped objects and its affordability, this model was chosen in the study for conducting simulations.

2.3. Governing Differential Equations. The human thermal model to be investigated consists of three interactive systems, namely: (a) passive system: this includes the body tissues, organs, circulatory and respiratory systems; (b) control systems: this system represents the thermal control functions of the body; and (c) clothing system: this system takes into account the thermal functions of the clothing.

The development of the thermal model for the clothed human includes governing energy equations of these three systems. The human thermal responses are obtained solving these systems of energy equations. Auxiliary equations, such as the blood pressure governing equation of the macrocirculatory system, the humidity ratio equation of the respiratory system, mass transfer equation of the clothing system, and control system algebraic equations, form the complete system of governing equations. As the main focus of this study is to examine the thermodynamic aspects of human thermal activity, the details pertaining to the auxiliary equations are not provided in this paper. However, the readers are encouraged to refer to the detailed finite element formulation of the human thermal system provided in Smith [3] and $\mathrm{Fu}[4]$ in a concise manner. Only the thermal energy equations that contribute to the temperature distribution in the human body are considered in the presentation. There are three differential thermal energy equations governing the thermal stress response in the passive human system and they include tissue, blood, and air energy equations. The tissue energy equation is the most predominant that determines the temperature distribution in three-dimensional framework.

For a tissue element, the energy balance equation is expressed as

$$
\rho C \frac{\partial T}{\partial t}=\nabla \cdot k \nabla T+q_{b}+q_{m}+q_{a}+q_{v}+\propto q_{\mathrm{res}} .
$$

The term on the left-hand side of (1) is the rate of accumulation of thermal energy per unit volume due to changing temperature of the tissue. The quantity equals the sum of six terms on the right-hand side which are summarized as follows,

$\nabla \cdot k \nabla T=$ net rate of heat conduction into a unit volume.

$q_{b}=\rho_{b} w_{b} C_{p, b}\left(T_{a}-T_{v}\right)=$ heat transfer rate into unit volume due to capillary blood perfusion.

$q_{m}=$ rate of thermal energy generation by metabolic reactions.

$q_{a}=2 \pi r_{a} h_{a}\left(T_{a}-T\right)=$ heat transfer rate from the blood in large arterial vessels to the tissue.

$q_{v}=2 \pi r_{v} h_{a}\left(T_{v}-T\right)=$ heat transfer rate from the blood in large arterial vessels to the tissue.

$q_{\text {res }}=2 \pi r_{\text {res }} h_{\text {res }}\left(T_{\text {res }}-T\right)+2 \pi r_{\text {res }} h_{\text {res }} \rho_{\text {da }} h_{\text {fg }}\left(W_{\text {air }}-\right.$ $\left.W_{\text {sat }}\right)=$ heat transfer rate from the respiratory system to the tissue. This term appears only in the head, neck, and torso where $\alpha=1$; for other parts of the body, $\alpha=0$.

2.4. Finite Element Human Thermal Model (FEHTM). In this study, the finite element method as described by Segerlind [20] is used to develop a solution procedure for solving 
the passive system governing equations along with other auxiliary equations. The solution domain for the passive system is discretized into a number of smaller elements. Each element represents a tissue section, blood vessel, respiratory tract, or portion of an internal organ. After the element mesh is generated, the passive system thermal governing equations along with the clothing thermal model and the environmental conditions are integrated for each element in terms of its parameters and unknown variables, yielding a system of simultaneous equations, which can then be solved for the unknown variables of interest.

The shape functions are derived for each different element shape. The quadratic shape functions are used to solve time-dependent field problems in this study. Thus, the linear shape functions are presented as follows.

(1) The shape functions for the triangular elements are:

$$
\begin{aligned}
& N_{1}=[r \theta z] /[\Delta r \Delta \theta \Delta z] ; \\
& N_{2}=[r(\Delta \theta-\theta) z] /[\Delta r \Delta \theta \Delta z] ; \\
& N_{3}=[(\Delta r-r) \Delta \theta z] /[\Delta r \Delta \theta \Delta z] ; \\
& N_{4}=[r \theta(\Delta z-z)] /[\Delta r \Delta \theta \Delta z] ; \\
& N_{5}=[r(\Delta \theta-\theta)(\Delta z-z)] /[\Delta r \Delta \theta \Delta z] ; \\
& N_{6}=[(\Delta r-r) \Delta \theta(\Delta z-z)] /[\Delta r \Delta \theta \Delta z] .
\end{aligned}
$$

(2) The shape functions for the rectangular elements are:

$$
\begin{aligned}
& N_{1}=\left[\left(r-r_{o}\right) \theta z\right] /[\Delta r \Delta \theta \Delta z] ; \\
& N_{2}=-\left[\left(r-r_{o}\right)(\theta-\Delta \theta) z\right] /[\Delta r \Delta \theta \Delta z] ; \\
& N_{3}=\left[\left(r-\left(r_{o}+\Delta r\right)\right)(\theta-\Delta \theta) z\right] /[\Delta r \Delta \theta \Delta z] ; \\
& N_{4}=-\left[\left(r-\left(r_{o}+\Delta r\right)\right) \theta z\right] /[\Delta r \Delta \theta \Delta z] ; \\
& N_{5}=-\left[\left(r-r_{o}\right) \theta(z-\Delta z)\right] /[\Delta r \Delta \theta \Delta z] ; \\
& N_{6}=\left[\left(r-r_{o}\right)(\theta-\Delta \theta)(z-\Delta z)\right] /[\Delta r \Delta \theta \Delta z] ; \\
& N_{7}=-\left[\left(r-\left(r_{o}+\Delta r\right)\right)(\theta-\Delta \theta)(z-\Delta z)\right] / \\
& {[\Delta r \Delta \theta \Delta z] ;} \\
& N_{8}=\left[\left(r-\left(r_{o}+\Delta r\right)\right) \theta(z-\Delta z)\right] /[\Delta r \Delta \theta \Delta z] .
\end{aligned}
$$

(3) The shape functions for the one-dimensional elements are:

$$
\begin{aligned}
& N_{1}=[\Delta z-z] / \Delta z \\
& N_{2}=z / \Delta z
\end{aligned}
$$

The Galerkin's method of weighted residuals is used to discretize governing equations, which have first order derivatives. The Galerkin's residual integral on the differential equations is evaluated with respect to the spatial coordinate(s) for each of the elements. The shape functions are used as weighting functions in the integral. The assembly of the resulting Galerkin's formulations is used to solve for the thermal responses of the passive system. Assembling these element equations by the direct stiffness method, a system of $N$ linear first order differential equations in terms of $N$ unknown temperatures and their rates of change is presented as

$$
[C]\left[\frac{d T}{d t}\right]^{T}+[K][T]^{T}=[F]^{T}
$$

where $[C]$ is the capacitance matrix, $[K]$ is the stiffness matrix, $[T]^{T}$ is the temperature vector, and $[F]^{T}$ is the forcing vector. By applying the central difference method to (4), the following equation is obtained:

$$
\begin{aligned}
\left([C]+\frac{\Delta t}{2}[K]\right)[T]_{t+\Delta t}^{T}= & \left([C]-\frac{\Delta t}{2}[K]\right)[T]_{t}^{T} \\
& +\frac{\Delta t}{2}\left([F]_{t}^{T}+[F]_{t+\Delta t}^{T}\right) .
\end{aligned}
$$

Equation (3) can be rewritten in the simplified form as

$$
[A][T]_{t+\Delta t}^{T}=[P][T]_{t}^{T}+\left[F^{*}\right],
$$

where $\Delta t$ is the time step size. Equation (3) is a system of linear simultaneous equations, which is solved by the computer for the nodal temperatures at time $t+\Delta t$.

The clothing system equations are solved using finite difference techniques and they are interfaced with the main model to determine the thermal responses of the clothed human. The readers who are interested to know the rest of the numerical solution details should refer to Segerlind [20]. The output from the FEHTM is used as input to the entropy generation model (EGM).

\subsection{Initial and Boundary Conditions}

2.5.1. Initial Conditions. The thermoneutral conditions are used as initial conditions for the human body. Instead of just the core and skin temperatures, the initial thermoneutral temperature distribution of the whole human body is needed because of the finite elements used in the body. In order to obtain this initial condition, the model is simulated for a nude sedentary human body starting with an approximate initial thermo-neutral temperature distribution. The air temperature and relative humidity used in the simulation are $28^{\circ} \mathrm{C}$ and $32 \%$. The temperature thus obtained is used as the initial condition for all other simulations. The initial condition for the clothing system is $\mathrm{Pi}$ and $\mathrm{Ti}$ for each clothing layer element.

2.5.2. Boundary Conditions. The boundary conditions required are the temperature $T$ and the vapor pressure $P$ of the surroundings. If a skin area is covered by clothing, the values of $T$ and $P$ of the inner most clothing layer become the required boundary conditions. Otherwise, the values of $T$ and $P$ of the environment become the required boundary conditions.

2.6. Entropy Generation Calculations. The only thermodynamic quantity that would characterize the whole humanthermal environment by including both environmental and physiological variables is entropy generation. The entropy generation provides a global measure of violent motions and reactions occurring in nature. Hence, the entropy generation in the human thermal system shows the extent of activeness of (1) thermal energy flows and (2) motions and reactions of substances within the body as a whole [1]. Therefore, the entropy generation is a significant thermodynamic quantity 
that characterizes the combined human-thermal environment from a holistic viewpoint. The entropy generation $\left(S_{\text {gen }}, \mathrm{J} / \mathrm{sec} / \mathrm{K}\right)$ is expressed as follows:

$$
S_{\text {gen }}=f\{\text { Physiology, Thermal Environment }\} .
$$

The following physical variables are categorized as follows to compute entropy generation.

\section{(i) Thermal Environmental Variables}

Thermal resistance of the clothing $\left(I_{\mathrm{cl}}, \mathrm{clo}\right)$;

air temperature $\left(T_{\text {air }}, \mathrm{K}\right)$;

relative humidity ( $\mathrm{RH}, \%)$;

activity Level or metabolic heat generation ( $M$, Joules/hr- $\mathrm{cm}^{2}$ ).

\section{(ii) Physiological Responses}

Skin temperature $\left(T_{\text {skin }}, \mathrm{K}\right)$;

core temperature $\left(T_{\text {core }}, \mathrm{K}\right)$;

convective heat loss from the skin surface $\left(E_{\mathrm{CNV}}\right.$, Joules/sec);

radiative heat loss from the skin surface ( $E_{\mathrm{RAD}}$, Joules/sec);

evaporative heat loss from the skin surface ( $E_{\mathrm{EVAP}}$, Joules/sec);

convective heat loss due to respiration ( $E_{\mathrm{RES}} \mathrm{CNV}$, Joules/sec);

evaporative heat loss due to respiration (ERESEVAP, Joules/sec).

The human entropy generation term is derived from the second law of thermodynamics. However, entropy generation $\left(S_{\text {gen }}, \mathrm{J} / \mathrm{sec} / \mathrm{K}\right)$ is found to be a function of both environmental variables and human thermal responses, that is,

$$
\begin{aligned}
S_{\text {gen }}=f & \left\{T_{\text {skin }}, T_{\text {core }}, E_{\mathrm{CNV}}, E_{\mathrm{RAD}}, E_{\mathrm{EVAP}},\right. \\
& \left.E_{\text {RES_CNV }}, E_{\text {RES_EVAP }}, M, I_{\mathrm{cl}}, T_{\text {air }}, \mathrm{RH}\right\} .
\end{aligned}
$$

Living organisms including human beings belong to the class of open systems. This is due to the fact that they have to continuously exchange energy and matter with the surroundings in order to maintain homeostasis, which is essential for the existence and support of life. Nicolis and Prigogine [21] formulated an extended version of the second law of thermodynamics applicable to both closed and open systems.

Consider the entropy change $d S$ during a time interval $d t$. The entropy change $d S$ is decomposed into sum of two contributions as expressed:

$$
d S=d S_{\text {flow }}+d S_{\text {gen }} .
$$

In the present study, (5) is modified as shown by Aoki [1, 2] as

$$
\Delta S=S_{\text {flow }}+S_{\text {gen }}
$$

where $\Delta S=$ total entropy change, $S_{\text {flow }}=$ net entropy flow, and $S_{\text {gen }}=$ entropy generation

From (6), the entropy generation in the human body is given by

$$
S_{\text {gen }}=\Delta S-S_{\text {flow }}
$$

where $\Delta S=$ total thermal energy generation in the body $(Q) /$ core temperature $\left(T_{\text {core }}\right)$ :

$$
\begin{aligned}
S_{\text {flow }}=S_{\text {in }}-( & S_{\text {out }}+S_{\mathrm{CNV}}+S_{\mathrm{EVAP}}+S_{\mathrm{RAD}} \\
& \left.+S_{\mathrm{CNV} \_ \text {RES }}+S_{\text {EVAP_RES }}\right) .
\end{aligned}
$$

The terms $S_{\text {in }}$ and $S_{\text {out }}$ on the right side of (10) are given as follows:

$$
\begin{aligned}
S_{\text {in }} & =2.05 \sigma\left(T_{\text {air }}\right)^{3}, \\
S_{\text {out }} & =2.05 \sigma\left(T_{\text {skin }}\right)^{3},
\end{aligned}
$$

where $\sigma=$ Stefan-Boltzmann constant $=5.67 \times$ $10^{-8} \mathrm{~J} \cdot \mathrm{m}^{-2} \mathrm{~s}^{-1} \mathrm{~K}^{-4}$. As this analysis is conducted for human thermal system in an indoor environment, the solar radiation is considered to be negligible and is not considered in this analysis. The other terms are:

$$
\begin{aligned}
S_{\mathrm{CNV}} & =\frac{E_{\mathrm{CNV}}}{T_{\text {skin }}}, \\
S_{\mathrm{RAD}} & =\frac{E_{\mathrm{RAD}}}{T_{\text {skin }}}, \\
S_{\mathrm{EVAP}} & =\frac{E_{\mathrm{EVAP}}}{T_{\text {core }}}, \\
S_{\mathrm{CNV} \_ \text {RES }} & =\frac{E_{\mathrm{CNV} \_\mathrm{RES}}}{T_{\text {skin }}}, \\
S_{\text {EVAP_RES }} & =\frac{E_{\text {EVAP_RES }}}{T_{\text {core }}} .
\end{aligned}
$$

Substituting the results of (11)-(12) into (9), the entropy generation is computed.

2.7. Using FEHTM Simulation Output to Compute Entropy Generation. As shown in Figure 1, the entropy generated in the human-thermal environmental system is interpreted as a quantitative measure of human thermo-physiological response to internal and external changes. The simulation results from the Finite-Element Human Thermal Model (FEHTM) are used as input to the entropy generation expression, which in turn provides Human Entropy Generation (HEG) values under different environmental conditions. 


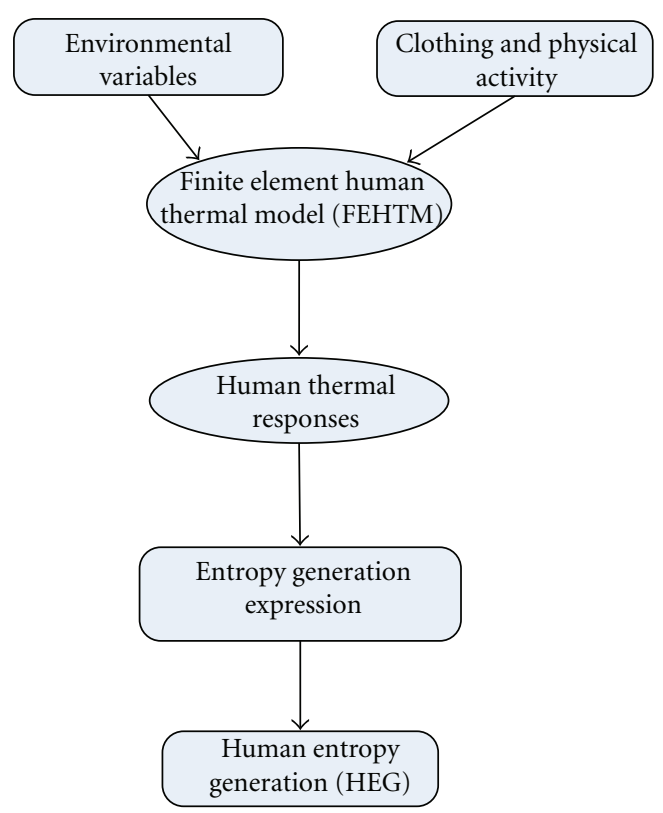

Figure 1: Evaluating entropy generation in a human thermal system.

\section{Results and Discussion}

3.1. Validation of Finite Element Human Thermal Model (FEHTM). The accuracy of the human thermal model is well-established through series of validations conducted in studies [3-5]. In these validation studies, the model predictions are compared with limited experimental data sources. The model is validated for variation of the core and skin temperatures, and latent skin heat loss by comparing with the experimental data of Hardy and Soderstrom [22]. Simulation is conducted for a 65 -minute at $286.15 \mathrm{~K}$ air temperature and $45 \%$ relative humidity. The comparison between experimental and model data for core body and mean skin temperatures are presented in Figures 2 and 3. The model predictions closely follow the experimental data. The average and maximum errors are $\pm 0.35^{\circ} \mathrm{C}$ and $\pm 0.54^{\circ} \mathrm{C}$ for core temperature, while $\pm 1.57^{\circ} \mathrm{C}$ and $\pm 4.42^{\circ} \mathrm{C}$ for mean skin temperature, respectively. In Figure 3, initially up to ten minutes, the deviation of model skin temperature from the experiment is around 3 to $4^{\circ} \mathrm{C}$. This is because the program starts the simulation using the thermoneutral conditions as the default initial conditions which are different from the experimental conditions. The predictions improve and follow the experimental data closely after this initial period of adjustment more in trend than in numerical accuracy. This is due to the fact that model depends on empirical relations to simulate human thermal control mechanisms or thermoregulation. The trigger for starting sweating (getting rid of excess heat) or shivering (production of heat) response is controlled by a set of empirical relations. The role of nervous system comprising of hypothalamus-pituitary-axis in controlling thermal response is the least understood mechanism, which makes it very difficult for the model to capture individual differences in thermal responses. This

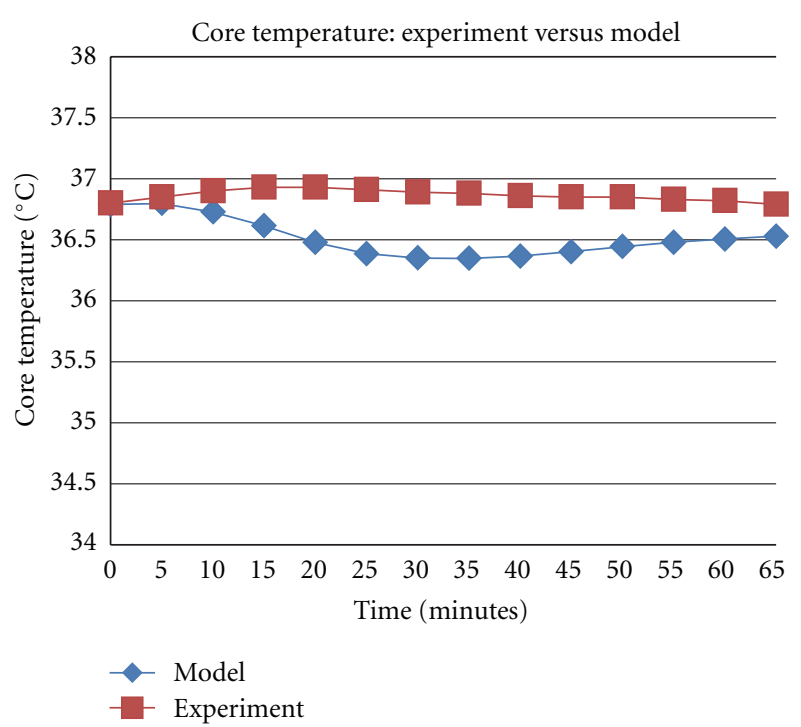

FIgURE 2: Core temperature versus time. Experimental data taken from Hardy and Soderstrom [22].

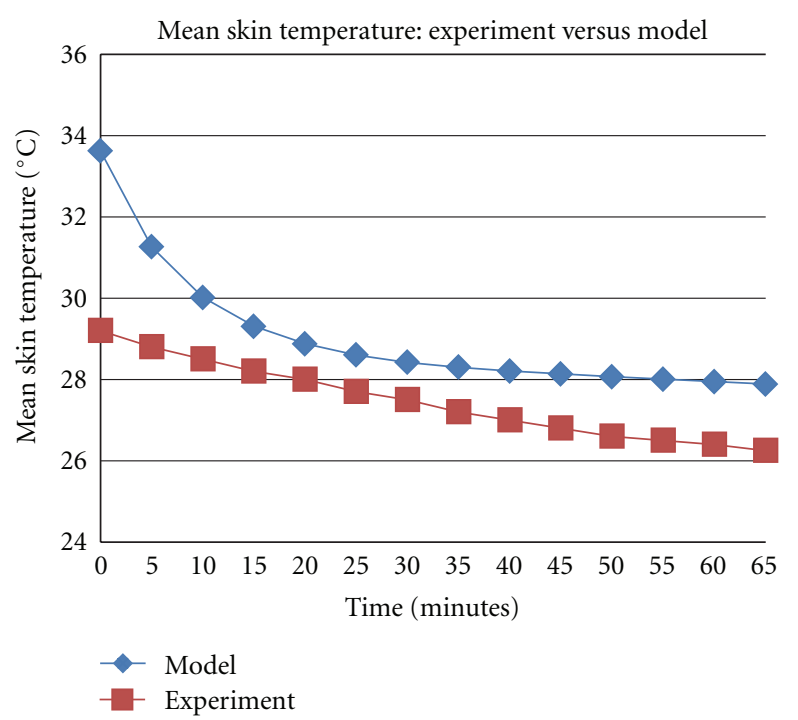

Figure 3: Mean skin temperature versus time. Experimental data taken from Hardy and Soderstrom [22].

will remain a major challenge until the thermoregulation is properly understood. Furthermore, the availability of experimental data is limited in the literature as it is expensive and risky to conduct these human thermal studies. Based on discussion in Section 2.2 on review of human thermal models pertaining to limitations of human thermal models, a significant effort is required to obtain data for any analytical study. In this regard, authors choose the best available model to obtain simulated data to demonstrate second-law application.

3.2. Effect of Physical and Personal Variables on Human Entropy Generation. The variables that affect overall thermal stress response include both physical and personal. 
The present study investigates the influence of four variables: air temperature, relative humidity, physical activity, and clothing on human entropy generation. Computer simulations were conducted and the impact of these external variables on entropy generation is computed. The initial conditions include air temperature and relative humidity at $28^{\circ} \mathrm{C}$ and $32 \%$ respectively. Figure 4 shows the variation of human entropy generation at lower RH of $30 \%$ for different air temperatures. The results indicate a lower value for higher air temperature $\left(T_{\text {air }}=310 \mathrm{~K}\right)$ and higher value for lower air temperature $\left(T_{\text {air }}=280 \mathrm{~K}\right)$. The decrease in ambient temperature causes a thermal response that leads to increase in thermal energy production within the body to maintain thermal homeostasis as explained in Section 2.1 earlier. This is clearly evident from the expression for entropy change $\left(\Delta S=Q / T_{\text {core }}\right)$ in $(9)$. The ambient temperature, at $37^{\circ} \mathrm{C}$, is higher than average mean skin temperature of $33^{\circ} \mathrm{C}$. This temperature difference of $4^{\circ} \mathrm{C}$ between body surface and the ambient triggers heat rejection from the skin surface thus removing any excess thermal energy produced in the core of the body via intervening tissues and organs. This decrease in thermal energy production $(Q)$ leads to reduction in entropy change $(\Delta S)$, which in turn causes a reduction in entropy generation $\left(S_{\text {gen }}\right)$ as per $(9)$. On the other hand, if the ambient environment continues to decrease below mean skin temperature, then this temperature difference will trigger shivering response leading to internal metabolic heat generation, which in turn causes entropy generation to increase as evident in Figure 4. In other words, the sweat response (due to higher ambient temperature) removes thermal energy produced in the body while the shivering response (due to lower ambient temperature) increases thermal energy production in the body. Both these processes are essential to keep the internal body temperature constant at $37^{\circ} \mathrm{C}$. Although human thermal stress increases under both conditions of hot and cold; however, it is beyond the scope of this paper to interpret entropy generation as a direct of measure of stress or a psychological variable such as perception.

As mentioned in Grandjean [23], it can be assumed that between $18^{\circ} \mathrm{C}$ and $24^{\circ} \mathrm{C}$ the relative humidity can fluctuate between $30 \%$ and $70 \%$ without creating thermal discomfort. This is clearly demonstrated in Figure 5 that the effect of changing relative humidity in the range of $30 \%$ and $70 \%$ relative humidity on entropy generation is insignificant. However, the effect of relative humidity at extreme low and higher air temperatures are presented in Figure 5, in which one can see the interaction effects of air temperature and relative humidity on human entropy generation response.

The physical activity level of a human being is described quantitatively as the metabolic free energy production " $M$ " expressed per unity body surface area. Some of this energy may be used to perform external work, but most is lost as thermal energy from the body. The metabolic rate is estimated by measuring the rate of oxygen consumption, which is considered to be constant for each simulation. A great deal of information is available in the literature on the metabolic rate associated with different physical activities [24]. Human thermal stress response largely depends on

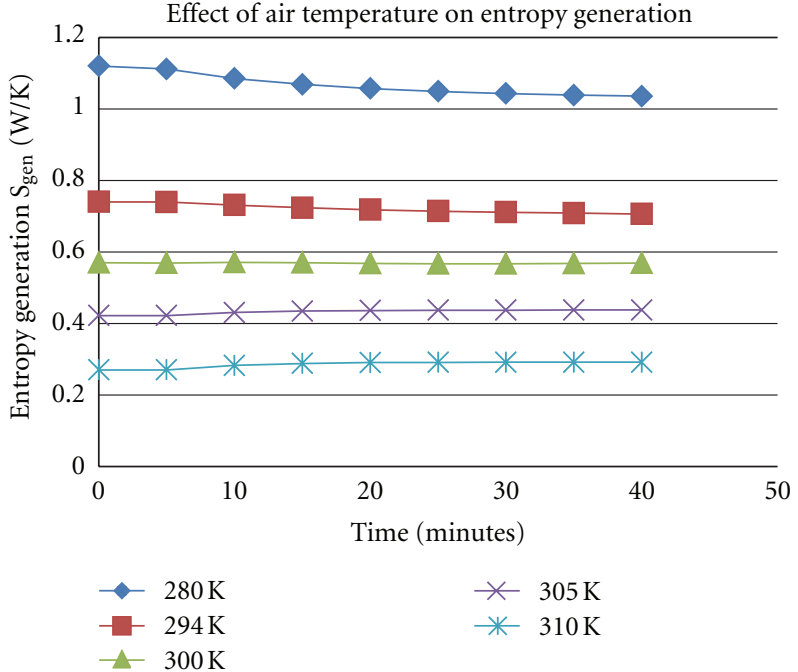

FIGURE 4: Effect of air temperature on entropy generation at $\mathrm{RH}=$ $30 \% ; M=21 \mathrm{~J} / \mathrm{hr}-\mathrm{cm}^{2}$; and $I_{\mathrm{cl}}=0.69$ clo.

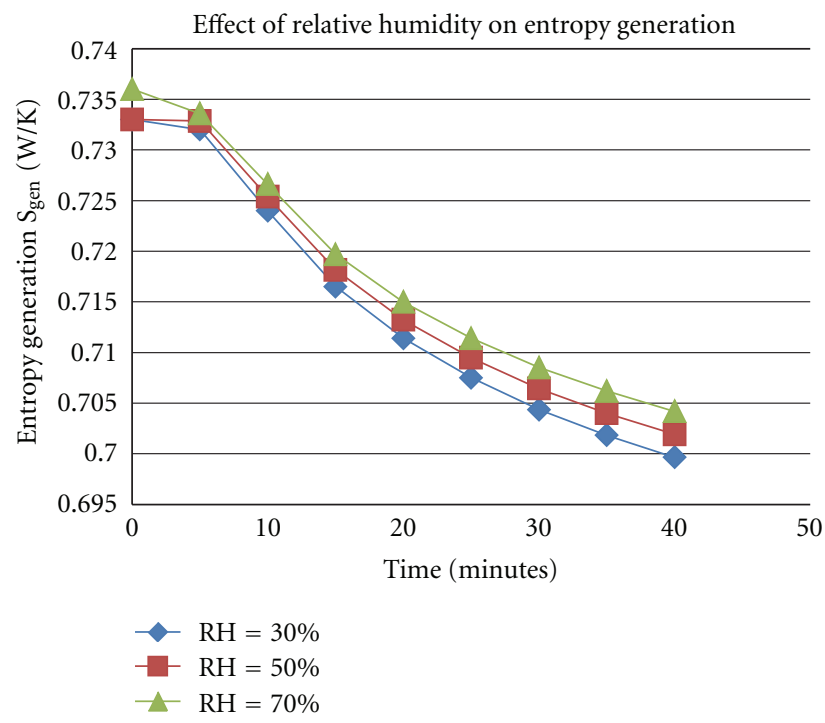

FIGURE 5: Effect of relative humidity on entropy generation at $T_{\text {air }}=$ $294 \mathrm{~K} ; M=21 \mathrm{~J} / \mathrm{hr}^{-\mathrm{cm}^{2}}$; and $I_{\mathrm{cl}}=0.69$ clo.

the type and intensity of work performed [25]. Physical work in the cold may lead to increased heat production and hence to decreased sensitivity to cold environment, while in the heat, hard physical work could be highly detrimental to the attainment of energy balance which could lead to hyperthermia. However, change in levels of physical activity has significant effect on the sensitivity of entropy generation as shown in Figure 6. The entropy generation increases with the decrease in physical activity as thermal energy produced in the body remains trapped due to low levels of thermal transport mechanisms contributing to increase in internal heat production. In other words, increase in physical activity leads to dissipation of thermal energy produced within the body and thus causing decrease in entropy generation. 


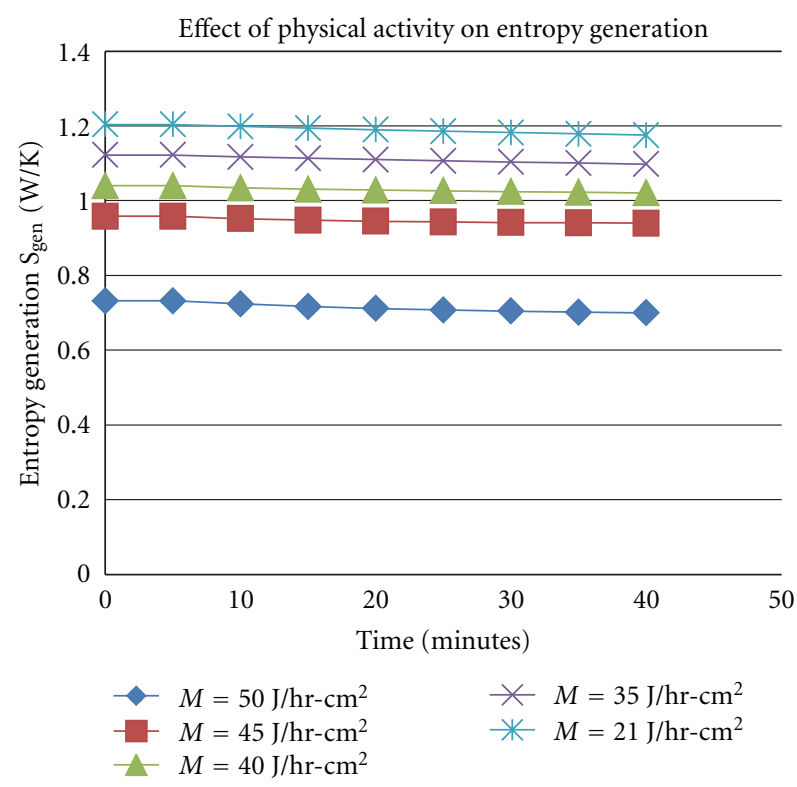

Figure 6: Effect of physical activity $\left(M, \mathrm{~J} / \mathrm{hr}-\mathrm{cm}^{2}\right)$ on entropy generation at $T_{\text {air }}=294 \mathrm{~K} ; \mathrm{RH}=30 \%$; and $I_{\mathrm{cl}}=0.69$ clo.

Human wear clothes under normal conditions. The transfer of thermal energy and mass (moisture) from skin through the clothing to the environment is an important factor. Clothing insulation is usually described as a single equivalent uniform layer over the whole body. Its insulating value is expressed in terms of "clo" units, defined as 1 clo $=0.155 \mathrm{~m}^{2} \cdot{ }^{\circ} \mathrm{C} / \mathrm{W}[26]$. The effect of clothing on entropy generation using three kinds of clothing which include light clothing with a thermal resistance value of $I_{\mathrm{cl}}=0.21 \mathrm{clo}$; medium clothing with $I_{\mathrm{cl}}=0.69$ clo; and heavy clothing with $I_{\mathrm{cl}}=1.72$ clo. The purpose of this simulation is to obtain quantitative data describing the dynamic nature of heat and moisture humans dissipate to their surroundings and to investigate entropy generation response for various types of clothing. The simulation was conducted for a period of forty minutes for constant environmental conditions of $294 \mathrm{~K}$ air temperature, $30 \%$ relative humidity and a physical activity level corresponding to a resting condition $(M=$ $21 \mathrm{~J} / \mathrm{hr}^{-} \mathrm{cm}^{2}$ ). With the skin area covered by clothing, the values of $T$ and $P$ of the inner most clothing layer become the required boundary conditions. It is observed from Figure 7 that as the thermal resistance (clo value) increases from light $\left(I_{\mathrm{cl}}=0.21\right)$ to heavy clothing $\left(I_{\mathrm{cl}}=1.72\right)$, entropy generation increases and remains a relatively higher value throughout the 40-minute simulation.

3.3. Design of Experiments (DOE) Technique. The most important physical variables which influence the human thermal stress response are air temperature $\left(T_{\text {air }}\right)$ and relative humidity (RH) for a given clothing and physical activity. The physical activity is assumed to be sedentary corresponding to the resting condition (metabolic rate, $M=21 \mathrm{~J} / \mathrm{hr}-$ $\mathrm{cm}^{2}$ ). Also, the clothing worn by human is expected to be of medium thermal resistance which corresponds to

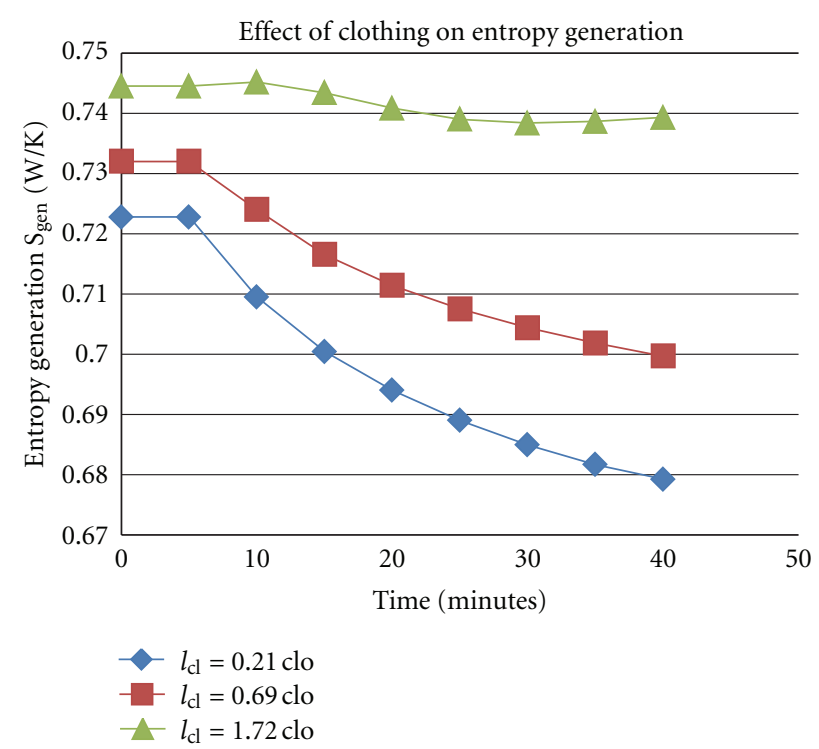

Figure 7: Effect of clothing on entropy generation at $T_{\text {air }}=294 \mathrm{~K}$; $\mathrm{RH}=30 \%$; and $M=21 \mathrm{~J} / \mathrm{hr}-\mathrm{cm}^{2}$.

a value of $I_{\mathrm{cl}}=0.69 \mathrm{clo}$. This, only two environmental variables that will be affecting entropy generation are air temperature and relative humidity. It would be interesting to observe the interaction effects of air temperature and relative humidity on entropy generation response. The technique of defining and investigating all possible conditions in an experiment involving multiple factors is known as the design of experiments $[27,28]$.

Consider a situation in which all other variables except air temperature and relative humidity have been fixed. The other fixed variable include moderate physical activity $(M=$ $\left.21 \mathrm{~J} / \mathrm{hr}-\mathrm{cm}^{2}\right)$ and clothing $\left(I_{\mathrm{cl}}=0.69\right)$. Each one of the four following different cases presented here is one of the simplest cases of design of experiments [28].

Case (i): Comfortable Environmental Conditions $\left(T_{\text {airl }}=\right.$ $294 \mathrm{~K} ; T_{\text {air } 2}=300 \mathrm{~K} ; \mathrm{RH}_{1}=40 \% ; \mathrm{RH}_{2}=60 \%$ )

Case (ii): Mild Environmental Conditions $\left(T_{\text {airl }}=290 \mathrm{~K}\right.$; $T_{\text {air } 2}=305 \mathrm{~K} ; \mathrm{RH}_{1}=30 \% ; \mathrm{RH}_{2}=70 \%$ )

Case (iii): Threshold Environmental Conditions $\left(T_{\text {airl }}=\right.$ $\left.280 \mathrm{~K} ; T_{\text {air } 2}=310 \mathrm{~K} ; \mathrm{RH}_{1}=20 \% ; \mathrm{RH}_{2}=80 \%\right)$

Case (iv): Extreme Environmental Conditions $\left(T_{\text {air }}=260 \mathrm{~K}\right.$; $\left.T_{\text {air } 2}=315 \mathrm{~K} ; \mathrm{RH}_{1}=10 \% ; \mathrm{RH}_{2}=90 \%\right)$

It involves two factors (air temperature and relative humidity) at two different levels (high and low) that affect the overall human thermal entropy generation. Two levels of air temperature, $T_{\text {air1 }}$ and $T_{\text {air } 2}$ and two levels of relative humidity, $\mathrm{RH}_{1}$, and $\mathrm{RH}_{2}$ are selected. (Subscripts 1 and 2, resp., refer to the low and high levels of each factor). Such an experiment is described as a $2 \times 2$ factorial experiment. There are four (22) possible treatments or combinations. For each one of these combinations of air temperature and 


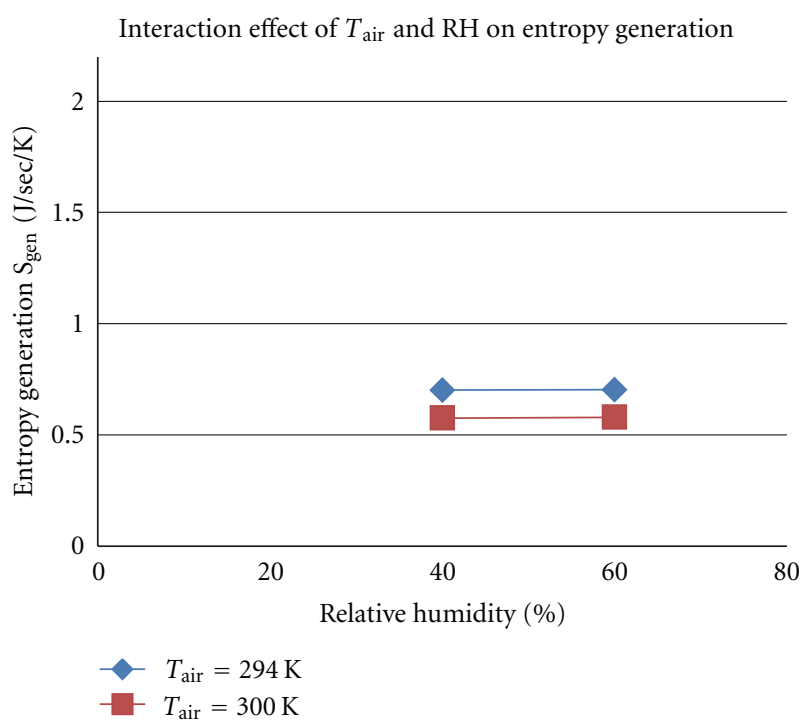

FIGURE 8: Entropy generation in comfortable environmental conditions.

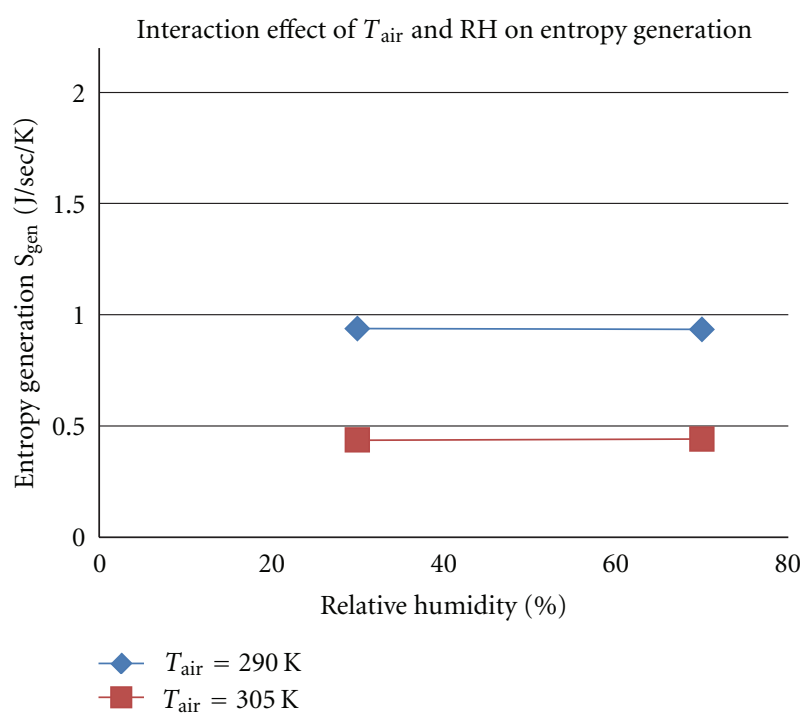

Figure 9: Entropy generation in mild environmental conditions.

relative humidity, a computer simulation is conducted, thus resulting in four simulations for each case and a total of twenty simulations for the entire demonstration of the DOE technique. Each simulation was conducted for one hour for a moderate physical activity $\left(M=21 \mathrm{~J} / \mathrm{hr}-\mathrm{cm}^{2}\right)$ and clothing $\left(I_{\mathrm{cl}}=0.69 \mathrm{clo}\right)$. The thermal responses at the end of the simulation (60th minute) were chosen for the computation of entropy generation. These thermal responses include core and mean skin temperatures, thermal energy losses, and energy production in the body. The human entropy

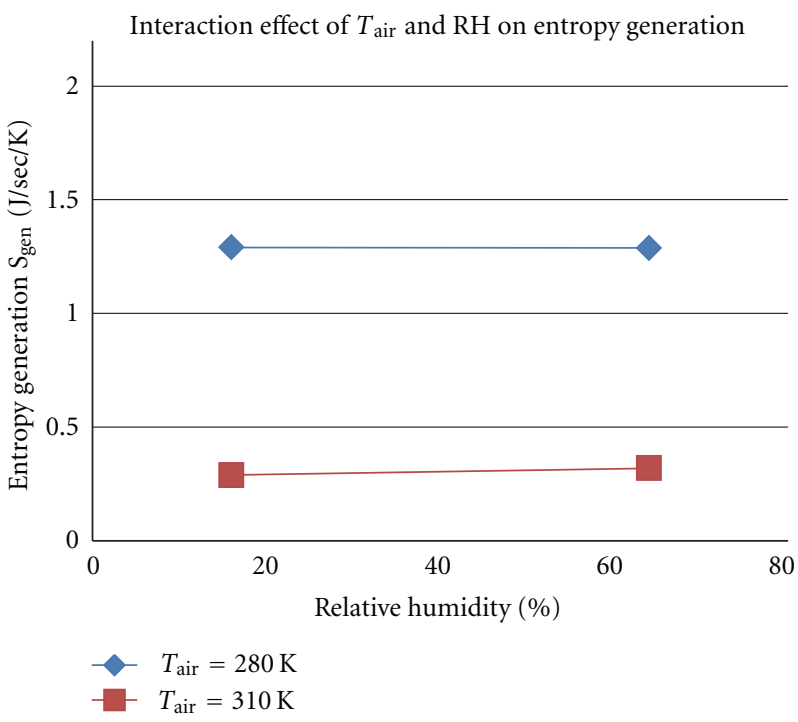

FIGURE 10: Entropy generation in threshold environmental conditions.

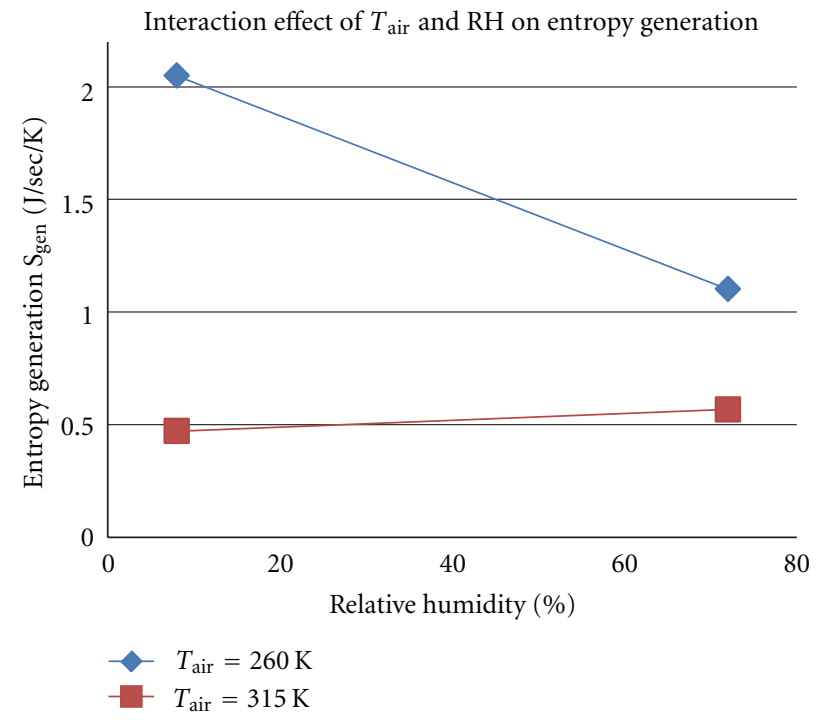

FIGURE 11: Entropy generation in extreme environmental conditions.

generation response to two factors ( $T_{\text {air }}$ and $\left.\mathrm{RH}\right)$ at two levels is given in Figures 8-11.

Case (i): Comfortable Environmental Conditions. As indicated in Figure 8, the comfortable environmental conditions range from $40 \%$ to $60 \% \mathrm{RH}$ and $294 \mathrm{~K}$ to $300 \mathrm{~K}$ air temperature. The entropy generation response values change slightly from 0.7005 to $0.7029 \mathrm{~J} / \mathrm{sec} / \mathrm{K}$ for $T_{\text {air }}=294 \mathrm{~K}$ and from 0.5756 to $0.5786 \mathrm{~J} / \mathrm{sec} / \mathrm{K}$ for $T_{\text {air }}=300 \mathrm{~K}$, respectively. The main effect of relative humidity is negligible, while that of air temperature is relatively visible from Figure 8. 
Case (ii): Mild Environmental Conditions. The mild environmental conditions range from $30 \%$ to $70 \% \mathrm{RH}$ and $290 \mathrm{~K}$ to $305 \mathrm{~K}$ air temperature. The entropy generation response values change slightly from 0.9374 to $0.9333 \mathrm{~J} / \mathrm{sec} / \mathrm{K}$ for $T_{\text {air }}=290 \mathrm{~K}$ and from 0.4362 to $0.4409 \mathrm{~J} / \mathrm{sec} / \mathrm{K}$ for $T_{\text {air }}=$ $305 \mathrm{~K}$, respectively. The main effect of relative humidity is negligible, while that of air temperature is relatively visible from Figure 9.

Case (iii): Threshold Environmental Conditions. Figure 10 shows the human entropy generation response to threshold environmental conditions which lie within the comfort zone of the ASHRAE comfort chart [29]. A closer examination reveals that there is no change in entropy generation response when relative humidity increases from $20 \%$ to $80 \%$ for an air temperature of $280 \mathrm{~K}$. While, there is a slight increase in stress response from 0.2883 to $0.3184 \mathrm{~J} / \mathrm{sec} / \mathrm{K}$ for an air temperature of $310 \mathrm{~K}$. On the other hand, for a relative humidity of $20 \%$, the stress response decreases for increase in air temperature to $310 \mathrm{~K}$. The same trend is observed for a relative humidity of $80 \%$.

Case (iv): Extreme Environmental Conditions. As shown in Figure 11, the examination of human entropy generation $\left(S_{\text {gen }}\right)$ response shows a decrease in its value for the relative humidity level of $90 \%$ at the lower level of air temperature $(260 \mathrm{~K})$. But the entropy generation increases when a higher level of air temperature $(315 \mathrm{~K})$ is introduced. These increases are called the simple effects of relative humidity.

It is observed that these human entropy generation response numerical values increase as environmental factors ( $T_{\text {air }}$ and $\mathrm{RH}$ ) gradually shift from the comfort zone defined in ASHRAE comfort chart [29] to extreme conditions as demonstrated in Figures $8-11$. This trend indicates that entropy generation could be utilized to characterize the human-thermal environment interaction. In this regard, some of the widely used indices are: Effective Temperature (ET), Heat Stress Index (HSI), Wet-Bulb Globe Temperature (WBGT), Wind Chill Index (WCI), and Predicted Mean Vote (PMV), are presented by ASHRAE [29]. These indices are empirical in nature and cannot be extended to varying environmental conditions and do not take into account individual physiological difference. Although PMV indirectly uses mean skin temperature and sweat response variables in the formulation, it is difficult to use and implement in an operational or real-time environments [30]. The present entropy generation method overcomes most of the limitations of the earlier indices in providing an inferential measure of either thermal stress or comfort.

\section{Conclusion}

A novel thermodynamic approach is developed to characterize human thermal stress responses in different environmental conditions. Thermal stress responses required to compute entropy generation are obtained through computer simulation, which is performed with the aid of a finiteelement human thermal model (FEHTM). The model is validated against the experimental data in the earlier studies. A series of simulations are conducted to examine the influence of air temperature, relative humidity, physical activity, and clothing on entropy generation. A design of experiments (DOE) is implemented to analyze in particular, the effects of two environmental factors (air temperature and relative humidity) at two levels on entropy generation in the human thermal system. It has been observed that the human entropy generation response is quite sensitive to changes in environmental conditions. It provides a global measure of activeness or thermal discomfort or stress in body. It could become a valuable measure to determine the quality of thermal environments or characterize human-thermal environment interaction. The biggest challenge is to design a space enclosure that would produce a desirable entropy generation in majority of occupants thereby fulfilling the comfort requirements. Further, the entropy generation methodology could also be used in military human performance applications, rehabilitation or athletic training, and sports to optimize the physiological performance based on environmental and personal variables.

\section{Nomenclature}

$C_{p}$ : $\quad$ Specific heat or heat capacity, $\mathrm{J} / \mathrm{g}-{ }^{\circ} \mathrm{C}$

$E_{\mathrm{CNV}}$ : Convective heat loss (J/s)

$E_{\text {EVAP: }} \quad$ Evaporative heat loss (J/s)

$E_{\mathrm{RAD}}: \quad$ Radiative heat loss $(\mathrm{J} / \mathrm{s})$

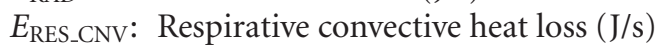

$E_{\text {RES_EVAP: }}$ Respirative evaporative heat loss (J/s)

$h$ : $\quad$ Surf. heat transfer coeff., J/hr- ${ }^{\circ} \mathrm{C}-\mathrm{cm}^{2}$

$h_{f g}: \quad$ Latent heat of vaporization, $\mathrm{J} / \mathrm{g}$

$I_{\mathrm{cl}}$ : $\quad$ Thermal resistance of the clothing (clo)

M: $\quad$ Metabolic activity $\left(\mathrm{J} / \mathrm{hr}-\mathrm{cm}^{2}\right)$

$P_{\text {vap: }} \quad$ Vapor pressure $(\mathrm{kPa})$

PMV: $\quad$ Predicted mean vote (dimensionless)

$q_{b}$ : Thermal energy rate into unit volume due to capillary blood perfusion

$q_{m}$ : $\quad$ Rate of thermal energy generation by metabolic reactions

$q_{a}$ : Thermal energy rate from the blood in large arterial vessels to the tissue

$q_{v}$ : $\quad$ Thermal energy rate from the blood in large venous vessels to the tissue

$q_{\text {res }}$ : Thermal energy rate from the respiratory system to the tissue. This term appears only in the head, neck, and torso where $\alpha=1$; for other parts of the body, $\alpha=0$

RH: $\quad$ Relative humidity (\%)

$S_{\text {gen }}$ : $\quad$ Entropy generation $(\mathrm{J} / \mathrm{sec} / \mathrm{K})$

$T: \quad$ Tissue temperature $(\mathrm{K})$

$T_{b}$ : $\quad$ Blood temperature (K)

$T_{\text {res }}$ : $\quad$ Respiratory tract temperature

$T_{\text {air }}: \quad$ Air temperature $(\mathrm{K})$

$T_{\text {core }}$ : Core temperature $(\mathrm{K})$

$T_{\text {skin }}$ : $\quad$ Skin temperature $(\mathrm{K})$. 


\section{Greek Symbols}

$\alpha: \quad$ Fraction of the total body mass in the skin node

$\rho:$ Tissue density, $\mathrm{g} / \mathrm{cm}^{2}$

$\rho_{b}$ : Blood density, $\mathrm{g} / \mathrm{cm}^{2}$

$\rho_{\text {res }}$ : Respiratory air density

$\sigma: \quad$ Stefan-Boltzmann const, $\mathrm{J} \cdot \mathrm{m}^{-2} \mathrm{sec}^{-1} \mathrm{~K}^{-4}$.

\section{Acknowledgments}

This work, in part, was supported by NASA Langley Research Center of Hampton, Virginia, through a Cooperative Agreement Grant NCC1-254 ending April 1999. The Grant was monitored by Dr. Alan T. Pope. Authors express their special thanks to Dr. Byron W. Jones, Chair and Professor of Mechanical Engineering, Kansas State University, for valuable suggestions and guidance.

\section{References}

[1] I. Aoki, "Entropy flow and entropy production in the human body in basal conditions," Journal of Theoretical Biology, vol. 141, no. 1, pp. 11-21, 1989.

[2] I. Aoki, "Effects of exercise and chills on entropy production in human body," Journal of Theoretical Biology, vol. 145, no. 3, pp. 421-428, 1990.

[3] C. E. Smith, A transient three-dimensional model of thehuman thermal system [Ph.D. thesis], Kansas State University, Manhattan, Kan, USA, 1991.

[4] G. Fu, A transient, three-dimensional mathematical model for clothed human [Ph.D. thesis], Kansas State University, Manhattan, Kan, USA, 1995.

[5] S. C. Boregowda and S. N. Tiwari, "Numerical investigation of thermal comfort using a finite element human thermal model," in Proceedings of the 8th Joint AIAA/ASME Thermophysics and Heat Transfer Conference, AIAA Paper No. 20022877, St. Louis, Mo, USA, 2002.

[6] E. P. Gyftopoulos and G. P. Beretta, Thermodynamics: Foundations and Applications, Dover Publications, New York, NY, USA, 2005.

[7] L. L. Langley, Homeostasis, Reinhold Book Corporation, New York, NY, USA, 1965.

[8] B. W. Jones, "Capabilities and limitations of thermal models for use in thermal comfort standards," Energy and Buildings, vol. 34, no. 6, pp. 653-659, 2002.

[9] B. Givoni and R. F. Goldman, "Predicting metabolic energy cost.," Journal of Applied Physiology, vol. 30, no. 3, pp. 429433, 1971.

[10] A. P. Gagge, J. A. J. Stolwijk, and Y. Nishi, "An effective temperature scale based on a simple model of human physiological regulatory response," ASHRAE Transactions, vol. 77, no. 1, pp. 247-262, 1971.

[11] J. A. J. Stolwijk, "A Mathematical Model of Physiological Temperature Regulation in Man," NASA Contractor Report NASA CR-1855, 1971.

[12] Y. Cheng, J. Niu, and N. Gao, "Thermal comfort models: a review and numerical investigation," Building and Environment, vol. 47, pp. 13-22, 2011.

[13] N. Z. Azer and S. Hsu, "The prediction of thermal sensation from a simple thermoregulatory model," ASHRAE Transactions, vol. 83, no. 1, pp. 88-124, 1977.
[14] E. H. Wissler, "Mathematical simulation of human thermal behavior using whole body models," in Heat Transfer in Medicine and Biology., A. Shitzer and R. C. Eberhart, Eds., vol. 1, pp. 325-373, Plenum Press, New York, NY, USA, 1985.

[15] B. W. Jones and Y. Ogawa, "Transient interaction between the human body and the thermal environment," ASHRAE Transactions, vol. 98, no. 1, pp. 189-195, 1992.

[16] D. Fiala, K. J. Lomas, and M. Stohrer, "A computer model of human thermoregulation for a wide range of environmental conditions: the passive system," Journal of Applied Physiology, vol. 87, no. 5, pp. 1957-1972, 1999.

[17] C. Huizenga, H. Zhang, and E. Arens, "A model of human physiology and comfort for assessing complex thermal environments," Building and Environment, vol. 36, no. 6, pp. 691699, 2001.

[18] R. McGuffin, R. Burke, C. Huizenga, Z. Hui, A. Vlahinos, and G. Fu, "Human thermal comfort model and manikin," SAE Technical Paper Series Paper No. 2002-01-1955, 2002.

[19] S. Tanabe, T. Tsuziki, K. Kimura, and S. Horikawa, "Numerical simulation model of thermal regulation of man with 16 body parts of evaluating thermal environment," in Summaries of Technical Papers of Annual Meeting, Architectural Institute of Japan, 1995.

[20] L. J. Segerlind, Applied Finite Element Analysis, John Wiley \& Sons, New York, NY, USA, 2nd edition, 1984.

[21] G. Nicolis and I. Prigogine, Self-Organization in Nonequilibrium Systems, John Wiley \& Sons, New York, NY, USA, 1977.

[22] J. D. Hardy and G. F. Soderstrom, "Heat loss from the nude body and peripheral blood flow at temperatures of $22^{\circ} \mathrm{C}$ to $35^{\circ}$ C," Journal of Nutrition, vol. 15, pp. 435-447, 1938.

[23] E. Grandjean, Fitting the Task to the Man, Taylor \& Francis, London, UK, 1981.

[24] D. A. McIntyre, "Design requirements for a comfortable environment," in Bioengineering, K. Cena and J. A. Clark, Eds., pp. 195-220, Elsevier Scientific Publishing Company, New York, NY, USA, 1981.

[25] K. H. E. Kroemer, H. B. Kroemer, and K. E. Kroemer-Elbert, Ergonomics: How to Design for Ease and Efficiency, Prentice Hall, Englewood Cliffs, NJ, USA, 1994.

[26] P. O. Fanger, Thermal Comfort, McGraw-Hill, New York, NY, USA, 1970.

[27] R. A. Fisher, Design of Experiments, Oliver and Boyd, Edinburgh, UK, 1951.

[28] R. Roy, A Primer on Taguchi Method, D. Van Nostrand Reinhold Company, New York, NY, USA, 1990.

[29] ASHRAE, ASHRAE Handbook of Fundamentals, Chapter 8, 2009.

[30] B. A. Hertig and H. S. Belding, "Evaluation and Control of Heat Hazards," in Temperature-Its Measurement and Control in Science and Industry, J. D. Hardy, Ed., vol. 3, pp. 347-355, Reinhold, New York, NY, USA, 1963. 

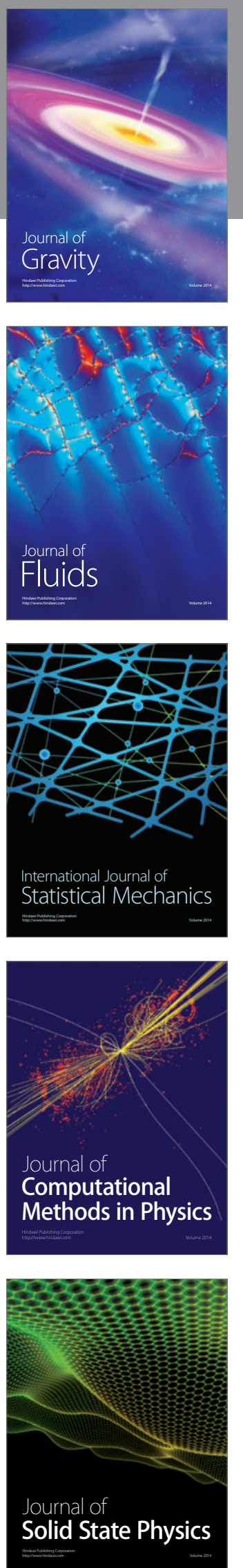
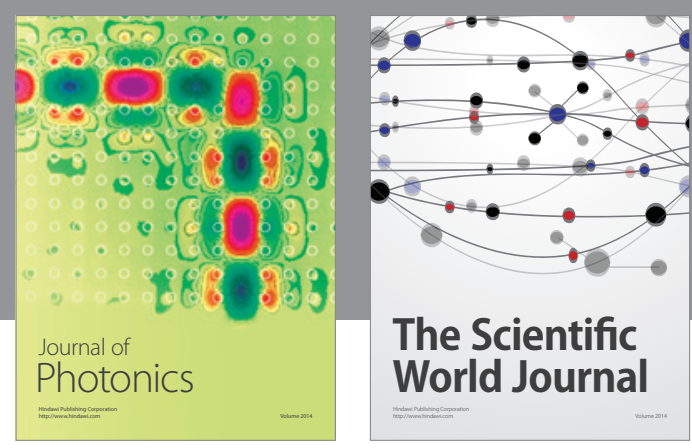

The Scientific World Journal

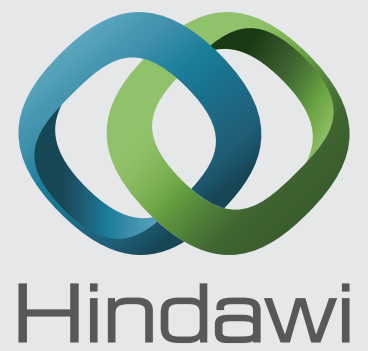

Submit your manuscripts at http://www.hindawi.com
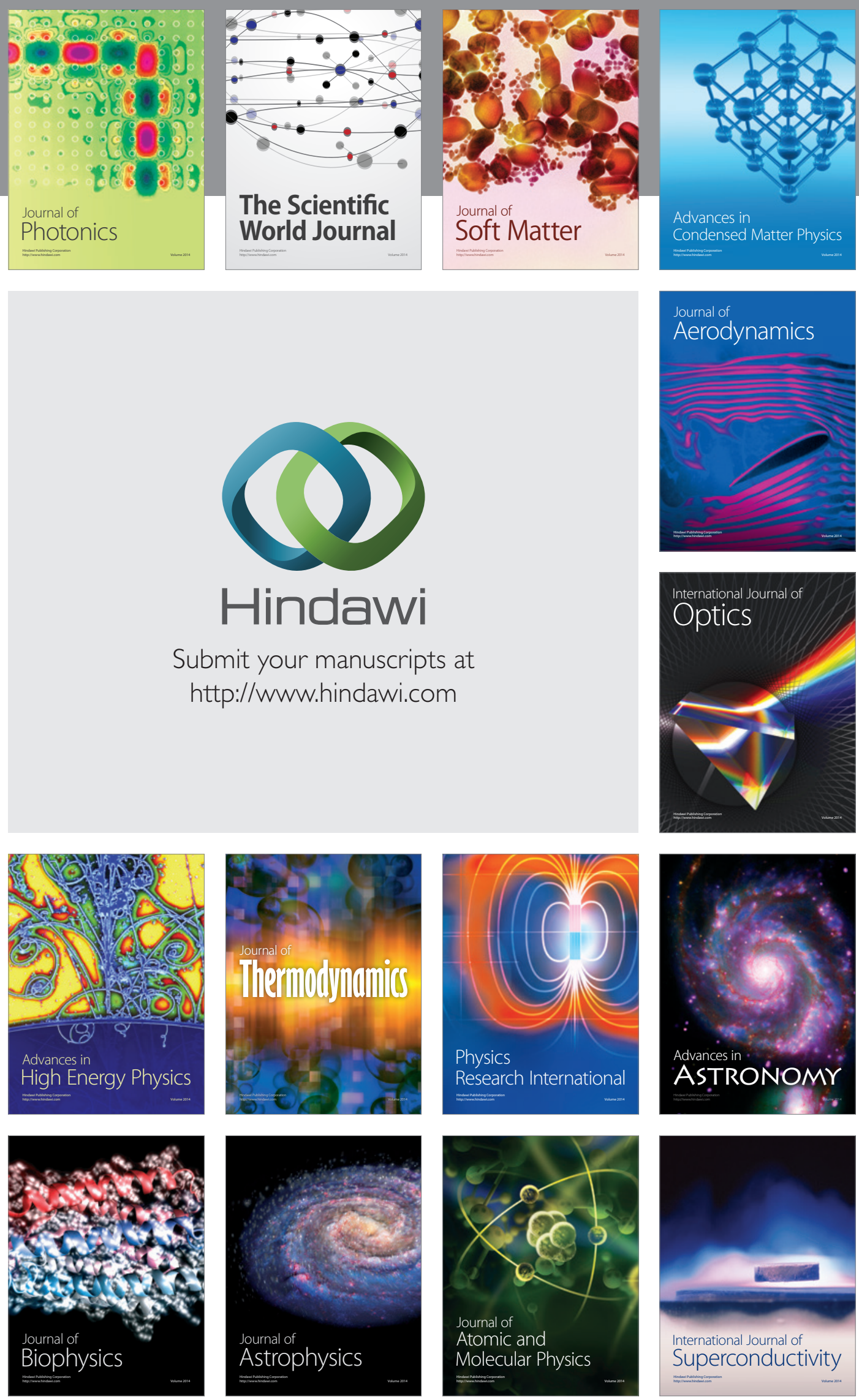
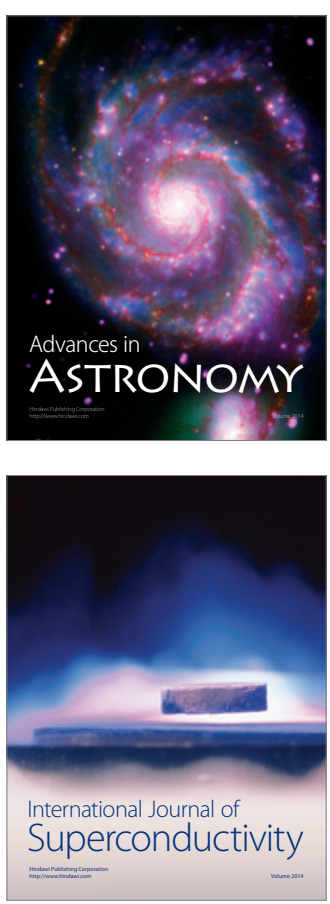